\title{
Catheter Related Blood Stream Infections: The Achilles's Heel of Hemodialysis Catheters.
}

\author{
Omer Sabir \\ Division of Nephrology \\ Department of Medicine \\ Fatima Memorial Hospital, Lahore, Pakistan
}

The vascular access in a hemodialysis patient is rightly considered to be the lifeline for the chronic kidney disease (CKD) patient. No doubt all the efforts are being put in to make an rteriovenous fistula acceptable to every CKD-V pre-dialysis patient slipping down the slope. ${ }^{1,2}$ In spite of this in our circumstances in Pakistan, every practising nephrologist knows in his heart that he will have to face clinical situations forcing the use of a temporary vascular access (TVA) in the form of double lumen (tunnelled/non tunnelled) hemodialysis catheters, on a daily basis. This impression holds true for developed countries where established registries give very high percentage of double lumen catheter use for initiation of hemodialysis. ${ }^{3}$ As far as the local situation is concerned, personal experience and published literature suggests that TVAs remain most frequently utilized since most of the dialysis are urgent/emergent start dialysis in our population. ${ }^{4,5}$

With TVAs, one has to accept the "unnecessary evil" : Catheter Related Blood Stream Infections (CRBSI). Infection of the hemodialysis catheter is an added burden on the healthcare systems in terms of cost, repeated hospitalization and mortality. ${ }^{6}$ The internationally reported figures coming from robust studies paint an unfavourable picture, although with a silver lining regarding adequate catheter care, however the local data is sparse. ${ }^{7,8}$ This lack of local data reflects partly upon, among other things, a deficient renal registry system. This month issue of PJKD carries an article by Mehmood SN et al that attempts to bridge this knowledge gap about the local prevalence of CRBSI in hemodialysis population. Previously published local literature suggests that the incidence is variable. ${ }^{10-14}$ The variable incidence may be a manifestation of different catheter protection protocols employed by different study centers, however its only recently that a consensus is formed regarding the proper reporting of catheter infections.

Mahmood SN et al shares their experience with CRBSI for both tunnelled-cuffed and non-tunnelled hemodialysis catheters. ${ }^{9}$ This differs from the previously published local literature where non tunnelled catheters are mainly used. Tunnelled catheters are expensive and require a more vigorous training for insertion and care, however, are known to be better than non-tunnelled catheters at prevention of CRBSI. The present study although gives similar rates of infections for both types of catheters which may reflect shorter insertion time hence less chances of intervention-driven infections and better post insertion care of non-tunnelled catheters. ${ }^{9}$ Previous studies either did not include tunnelled catheters or failed to report. ${ }^{9-14}$ It would have been enlightening to highlight the microbiological profiles with regards to the catheter type.

The present study stands out for reporting the microbiological profile of the CRBSI being encountered at the study centre. ${ }^{9}$ The profile is not dissimilar to the previously described local profile. ${ }^{9}$ Although it seems that gram positive organisms remain the main culprits, Mehmood SN et al give a more detailed breakdown of the incriminatory organisms which strengthens our knowledge about the magnitude of this problem. The presented data shows 59 out of 84 grown cultures were for gram positive organisms (70\%). This seems to be higher than the internationally reported figures, and may spur more vigilant insertion techniques and post insertion care protocols since gram positive organisms are likely to be carried into the tunnel (or the exit site for both tunnelled and non-tunnelled catheters) and catheter from the surrounding skin. ${ }^{16}$

The worrisome finding of high gentamicin resistance (61.5\%) should be a cause of concern for the local nephrology community. Gentamicin is cheap and easily available. Other options as described by Mahmood SN et al. may be more expensive for the local hemodialysis population. This trend of increased gentamicin resistance does not seem to be a local problem alone, however there is emerging data that the dosage of gentamicin used may have important implications for the emergence of resistance. ${ }^{17-19}$

It is time for the local nephrology community to revisit their practices regarding antibiotic lock solutions in the light of this commendable data by Mehmood SN so that maximum cost-effectiveness is maintained for the foreseeable future. The effort by Mahmood SN et al may be a springboard for further such studies to elucidate the existing patterns of CRBSI in local centres.'

\section{Corresponding Author}

Dr Omer Sabir,

MBBS, FCPS (Medicine \& Nephrology)

Department of Medicine

Division of Nephrology

Fatima memorial Hospital

Lahore, Pakistan

Email: omer.sabir@fmhcmd.edu.pk

\section{References}

1. Allon M: Current management of vascular access. Clin J Am Soc Nephrol 2007; 2: 786 - 800.

2. Brown RS, Patibandla BK, Goldfarb-Rumyantzev AS. The survival benefit of "fistula first, catheter last" in hemodialysis is primarily due to patient factors. J Am Soc Nephrol. 2017;28:645 - 652.

3. US Renal Data System: 2018 USRDS Annual Data Report: Volume 2: End-Stage Renal Disease in the United States, Bethesda, MD, National Institutes of Health, National Institute of Diabetes and Digestive and Kidney Diseases, 2018. Last accessed on July 19,2020.

4. Mahmood SN, Mukhtar KN, Iqbal N, Umair SF. Pre dialysis care and types of vascular access employed in incident hemodialysis patients: a study from Pakistan. Pak J Med Sci Sep 2013;29(3):828-31 
5. Sabir O, Riaz MM, Rafique K, Anjum N, Abbas G, Akram M. Fistula First-Are We There Yet? A Multicenter study. PJKD 2017;1(3):21-26.

6. Allon M. Vascular Access for Hemodialysis Patients New Data Should Guide Decision Making. CJASN 2019; 14 (6) 954-961.

7. Nguyen DB, Shugart A, Lines C, Shah AB, Edwards J, Pollock D, Sievert D, Patel PR: National healthcare safety network (NHSN) dialysis event surveillance report for 2014. Clin J Am Soc Nephrol 2017; 12: 1139 - 1146.

8. Fisher M, Golestaneh L, Allon M, Abreo K, Mokrzycki MH. Prevention of Bloodstream Infections in Patients Undergoing Hemodialysis. Clin J Am Soc Nephrol. 2020;15(1):132-151.

9. Mahmood SN, Asif S, Anwar MA, Naveed KO. Frequency and microbiological profile of catheter-related infections in hemodialysis patients receiving gentamicin as antimicrobial lock therapy for prophylaxis. Pak J Kidney Dis 2020;4(3):

10. Shaikh RA, Memon Q, Memon S, Rahopoto Q. Infective (non viral) complications of double lumen hemodialysis catheter in renal failure patients Med Channel 2005;11(2):46-8.

11. Sabir O, Tarif N, Rizwan S, Rafique K, Rizvi N, Khan A. Temporary hemodialysis; Taurolidine lock to prevent catheter-related blood stream infections. Professional Med J 2015;22(3):287-292.

12. Javaid B, Manzoor A, Elahi I, Javaid S. Catheter Related Infection (CRI): Comparison of Heparin Versus Citrate Annals Punjab Med Coll 2019;13(2):94-6.

13. Rafique K, Sabir O, Riaz MM, Rizvi N, Tarif N. Comparing the efficacy of Gentamicin-heparin solution to Heparin alone as catheter related blood stream infection in patients undergoing hemodialysis. Pak J Kidney Dis 2019;3(4):67-73.

14. Shahbaz M, Muhammad T, Umar. Prevention of catheter related blood stream infection using Heparin plus Gentamicin catheter lock solution in comparison to Heparin alone. A randomized controlled study. Pak J Kidney Dis 2019;3(4):59-66.

15. Allon M, Brouwer-Maier DJ, Abreo K, Baskin KM, Bregel K, Chand DH et al. Recommended clinical trial end points for dialysis catheters. Clin J Am Soc Nephrol 2018; 13: 495 - 500.

16. Kumbar L, Yee J. Current Concepts in Hemodialysis Vascular Access Infections. Adv Chronic Kidney Dis. 2019;26(1):16-22.

17. Landry DL, Braden GL, Gobeille SL, Haessler SD, Vaidya CK, Sweet SJ: Emergence of gentamicin-resistant bacteremia in hemodialysis patients receiving gentamicin lock catheter prophylaxis. Clin J Am Soc Nephrol 2010; 5: 1799 - 1804.

18. Moran J, Sun S, Khababa I, Pedan A, Doss S, Schiller B: A randomized trial comparing gentamicin/citrate and heparin locks for central venous catheters in maintenance hemodialysis patients. Am J Kidney Dis 2012; 59: 102 - 107.

19. Moore CL, Besarab A, Ajluni M, Soi V, Peterson EL, Johnson LE. Comparative effectiveness of two catheter locking solutions to reduce Catheter-Related Bloodstream Infection in hemodialysis patients. CJASN 2014; 9 (7) 1232-1239. 